\title{
Control of Shock-Wave/Bound-Layer Interactions by Bleed
}

\author{
T. I-P. Shih \\ Department of Aerospace Engineering, Iowa State University \\ Ames, Iowa, 50011-2271, U.S.A.
}

\begin{abstract}
Bleeding away a part of the boundary layer next to the wall is an effective method for controlling boundary-layer distortions from incident shock waves or curvature in geometry. When the boundary-layer flow is supersonic, the physics of bleeding with and without an incident shock wave is more complicated than just the removal of lower momentum fluid next to the wall. This paper reviews CFD studies of shock-wave/boundary-layer interactions on a flat plate with bleed into a plenum through a single hole, three holes in tandem, and four rows of staggered holes in which the simulation resolves not just the flow above the plate, but also the flow through each bleed hole and the plenum. The focus is on understanding the nature of the bleed process.
\end{abstract}

Keywords: shock-wave/boundary-layer interaction, inlet bleed.

\section{Introduction}

Shock-wave/boundary-layer interactions and their effective control play an important role in determining the successful operation of many aerodynamic and propulsion devices. Examples include mixed-compression inlets of turbojet engines, supersonic wind tunnels, and supersonic aircraft. For these devices, the adverse pressure gradient induced by the incident shock wave propagates upstream through the subsonic part of the boundary layer and can cause the flow to separate. Once flow separation takes place, the geometry of the device is changed and this can lead to severe consequences. For example, flow separation near the throat of a mixed-compression inlet can cause the "unstart" condition to occur and shut down the engine [1]. For a wind tunnel, flow separation creates flow distortion and reduces the effective Mach number in the test-section. For a supersonic aircraft, flow separation can increase drag, reduce lift, and lower the amount of air captured by the inlet for propulsion.

One effective way of controlling shock-wave induced, flow separation is to place bleed holes in the vicinity where the shock wave strikes the boundary layer. The ability of bleed to prevent separation for subsonic flow in adverse pressure gradients is well understood. For subsonic flow, bleed removes fluid near the wall, where the momentum is low because of no-slip so that the remaining higher momentum fluid can withstand the adverse pressure gradients without separating. For supersonic flow with and without incident shock waves, the mechanism of bleed is more complicated.

The objective of this paper is twofold. The first objective is to give an overview of the research on shock-wave/boundary-layer interactions with bleed. The second objective is to present results from numerical studies to illustrate the nature of the flow created by bleeding supersonic boundary layers on a flat plate into a plenum through holes with and without an incident shock wave.

\section{Overview}

The importance of bleed in controlling shock-wave/boundary-layer interactions has led a number of investigators to use both experimental and computational methods to study this problem (see reviews by Delery [2] and Hamed and Shang [3]). According to Hamed and Shang [3], though all experimental studies agree that bleed can control shock-wave/boundary-layer interactions, they disagree on how bleed-hole geometric and operating parameters influence the effectiveness of the bleed process. These discrepancies indicate the complexities of the flow in the region about bleed holes. In that region, many parameters can affect the flow with different ones dominating under different conditions.

In this overview, only work done after the review of Hamed and Shang [3] are discussed. Also, it will only include studies that address the details of the bleed process. To date, all such studies have interrogated shock-wave/boundary-layer interactions on a flat plate with bleed through either slots or circular holes. Unfortunately, there are very few experimental studies on this subject in the open literature. Willis, et al. [4,5] reported measurements of flow coefficients for a wide variety of bleed configurations for both choked and unchoked bleed. They also measured the profile of stagnation pressure in the boundary layer upstream and

Received January 1 2008; accepted for publication July 6 2008: Review conducted by Prof. Wu Yulin.

Corresponding author: Tom I-P. Shih, Professor, tomshih@iastate.edu

This manuscript was presented at The $9^{\text {th }}$ Asian International Conference on Fluid Machinery, October 16-19, 2007, Jeju - Korea. 
downstream of the bleed region. Bleed configurations studied include rows of circular holes arranged in a staggered fashion with various porosity and inclination of the hole relative to the approaching boundary-layer flow. The results of these experimental studies have been used to validate numerical studies.

Numerical studies on bleed reported so far are based on two- and three-dimensional (2- and 3-D) computational fluid dynamics (CFD) analyses. CFD studies on bleed in which the flow through each bleed hole is resolved have been reported by two research groups, one by Hamed and her co-workers and another by Shih, Chyu, and their co-workers. Hamed and co-workers (Hamed and Lehnig [6, 7] and Hamed, et al. [8,9]) focused on understanding bleed through a single slot via 2-D CFD analyses. Shih, Chyu, and their co-workers studied bleed through both slots and circular holes via 2-D CFD for slots and 3-D CFD for circular holes. Hahn, et al. [10] reported a study on bleed through single and multiple slots and examined the effects of spacing between slots, width/depth ratio of slots, and shock strength with focus on the minimum amount of bleed needed to eliminate shock-wave-induced flow separation. Rimlinger, et al. [11] and Shih, et al. [12] in a study involving bleed through a single normal hole and Chyu, et al. $[13,14]$ in a study involving bleed through three holes in tandem showed that bleed of a supersonic boundary layer induced the formation of a "barrier" shock in each hole and described the nature of that shock structure for normal and inclined holes with and without incident shock waves. Rimlinger, et al. [15-17], in a study involving four rows of bleed holes arranged in a staggered fashion, showed that two rows of staggered normal holes is able to eliminate shock-wave induced flow separation if the two rows are located just upstream of where the shock wave incidents on the boundary layer. Their computations were validated by comparing predicted CFD results with the measurements of Willis, et al. [4, 5]. Flores, et al. [18] studied the effects of misaligning the hole arrangement and the hole inclination angle with respect to the approaching flow. For normal (i.e., 90o) holes in a staggered arrangement, the worst case misalignment is the conversion of a staggered-hole arrangement to a nonstaggered-hole arrangement. For inclined holes, there is the added complication of turning of the supersonic flow, not just downwards into the plenum but also sideways. Their study showed such misalignments to strongly weaken the utility of bleed in controlling shock-wave-induced flow separation. Lin, et al. [19] studied the effects of plenum size on the bleed process. When the plenum is small, the bleed jets in the plenum interact and reduce the flow coefficients considerably. Lin, et al. [20] also studied passive bleed in which the high pressure downstream of the incident shock wave is used to drive a jet upstream of shock to increase the momentum of the fluid near the wall so that it can resist the adverse pressure gradient without separating. Their study examined several passive-bleed configurations. Though flow separation can be eliminated, flow distortions can still be significant because of the increased boundary-layer thickness.

For the CFD analyses, the generation of high-quality grid systems for bleed configurations with different arrangements of normal and inclined holes can be tedious and time consuming. Thus, Chyu, et al. [21] and Shih, et al. [22] developed a "knowledge-based" preprocessor, called AUTOMAT, to automate the grid generation process. Their preprocessor automatically generates all files needed to perform a CFD analysis of shock-wave/boundary-layer interactions on a flat plate with bleed through rows of holes including the grid system as well as initial and boundary conditions once geometric and operating parameters have been specified. Flores, et al. [23] further developed AUTOMAT to allow the study of bleed through micro holes in which the cross-section of the holes can vary along its length.

Since it is not feasible to resolve the flow through each bleed hole in practical configurations that may involve hundreds to thousands of holes connected to different plenums, it is important to develop "bleed" boundary conditions (BCs) over surfaces with bleed holes that model the key flow physics created by the bleed process [24]. Chyu, et al [25] developed and evaluated several bleed BCs for a mixed-compression inlet in which the bleed region was modeled as a porous surface. Reasonably good agreement with the experimental data of Smeltzer \& Sorensen [26] was found when the bleed through the holes was choked. Harloff \& Smith [27] developed a more comprehensive bleed BC by using the experimental data of Willis, et al. [4, 5]. The focus of the porous-type bleed BCs is to ensure that the bleed rate is modeled correctly, which is the most important part of the bleed process. However, by disregarding the bleed-hole geometry, some aspects of bleeding a supersonic boundary layer is lost. Paynter, et al. [28] and Lee, et al. [29] improved the porous-wall type of bleed BCs by adding a partial differential equation that resemble a one-equation turbulence model to model the roughness effects induced by the bleed process. Benson, et al. [30] presented a non-porous-wall bleed BC in which at least one grid point is within each bleed hole. With such a bleed BC, they showed that it is possible to get the bleed rate correct and account for some of the effects of the barrier shock. Benson, et al [31, 32] demonstrated the usefulness of the bleed BCs developed by Benson, et al. [30] for normal and inclined holes with and without incident shock waves and in an axisymmetric mixed-compression inlet.

\section{Bleed through Discrete Holes}

In this section, three bleed problems aimed at revealing the mechanisms of bleeding a supersonic boundary layer are described, formulated, and their numerical method of solution presented. In Section 4, the CFD results generated are presented.

\subsection{Problem Description}

To understand shock-wave/boundary-layer interactions on a flat plate with bleed through circular holes into a plenum, three bleed configurations are considered (see Fig. 1). In configuration 1, the bleed is through a single hole (Fig. 1(a) top). In configuration 2, the bleed is through three holes in tandem (Fig. 1(a) middle and bottom), which allows an examination on the effects of upstream and downstream holes. In configuration 3, the bleed is through four row of holes arranged in a staggered fashion (Fig. 1(c)), which represents a more realistic configuration and allows for an examination on the effects neighboring bleed holes - downstream, upstream, and on the sides. For all three bleed configurations, the holes can be normal (i.e., $\left.90^{\circ}\right)$ holes as shown in Fig. 1 or inclined (e.g., $20^{\circ}$ or $30^{\circ}$ relative to the freestream flow). Note that when a bleed hole is inclined, the cross section of that bleed hole in a plane parallel to the flat plate is an ellipse.

The domains of the three bleed configuration studied are the regions bounded by the dash and solid lines, which include the plenum, the region above the flat plate, and the bleed holes. For the configuration with four rows of bleed holes, only four "half" 
bleed holes were simulated because of symmetry in the spanwise direction. The dimensions in Fig. 1 are $\mathrm{W}=24 \mathrm{D}, \mathrm{H}=30 \mathrm{D}, \mathrm{L}_{\mathrm{P}}=$ 19D, $H_{P}=10 D$, and $H_{t}=D$ (Figs. 1(a) and 1(b)) and 2D (Fig. 1(c)), where D is the diameter of the bleed hole. D equals 0.003175 in Figs. 1 (a) and 1(b) and $0.00508 \mathrm{~m}$ in Fig. 1(c). In Fig. 1(c), $\theta$ is $60^{\circ}$ or $70^{\circ}$ and $\mathrm{L}_{\mathrm{y}}$ is $\mathrm{D}$ or $1.5 \mathrm{D}$.

For each of these three bleed configurations, the fluid that enters the domain above the flat plate is air with constant specificheats ratio $(\gamma)$ of 1.4 . The freestream Mach number $\left(\mathrm{M}_{\infty}\right)$, static temperature $\left(\mathrm{T}_{\infty}\right)$, and density $\left(\rho_{\infty}\right)$ are 1.6 or $2.5,134 \mathrm{~K}$, and 0.262 $\mathrm{kg} / \mathrm{m}^{3}$, respectively. At the inflow boundary, the thickness of the turbulent boundary layer $(\delta)$ is $0.003175 \mathrm{~m}$. Several back pressures $\left(\mathrm{P}_{\mathrm{b}}\right)$ at the exit of the plenum were imposed to study choked as well as unchoked bleed in the bleed holes: $0.3 \mathrm{P}_{\infty}$ to $1.7 \mathrm{P}_{\infty}$, where $\mathrm{P}_{\infty}$ is the freestream static pressure.

When there is an incident shock wave that impinges on the turbulent boundary layer, it is generated by an oblique shockwave generator. In all cases, the shock wave generated was strong enough to induce flow separation on the flat plate in the absence of bleed. Also, the shock wave impinged at a fixed location on the flat plate regardless of the angle of the bleed hole or the number of bleed holes.

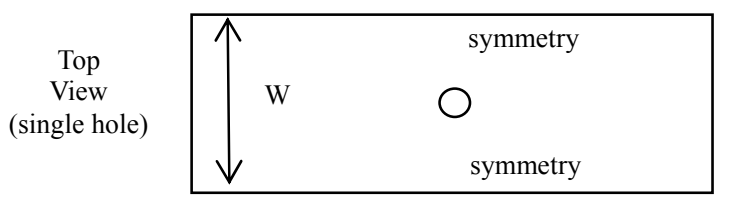

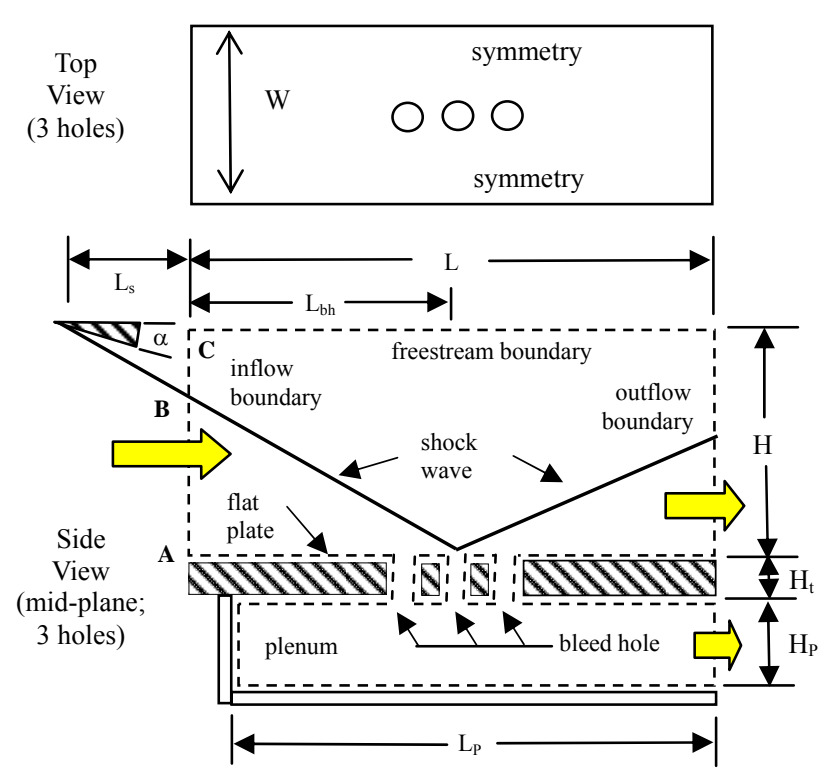

(a)
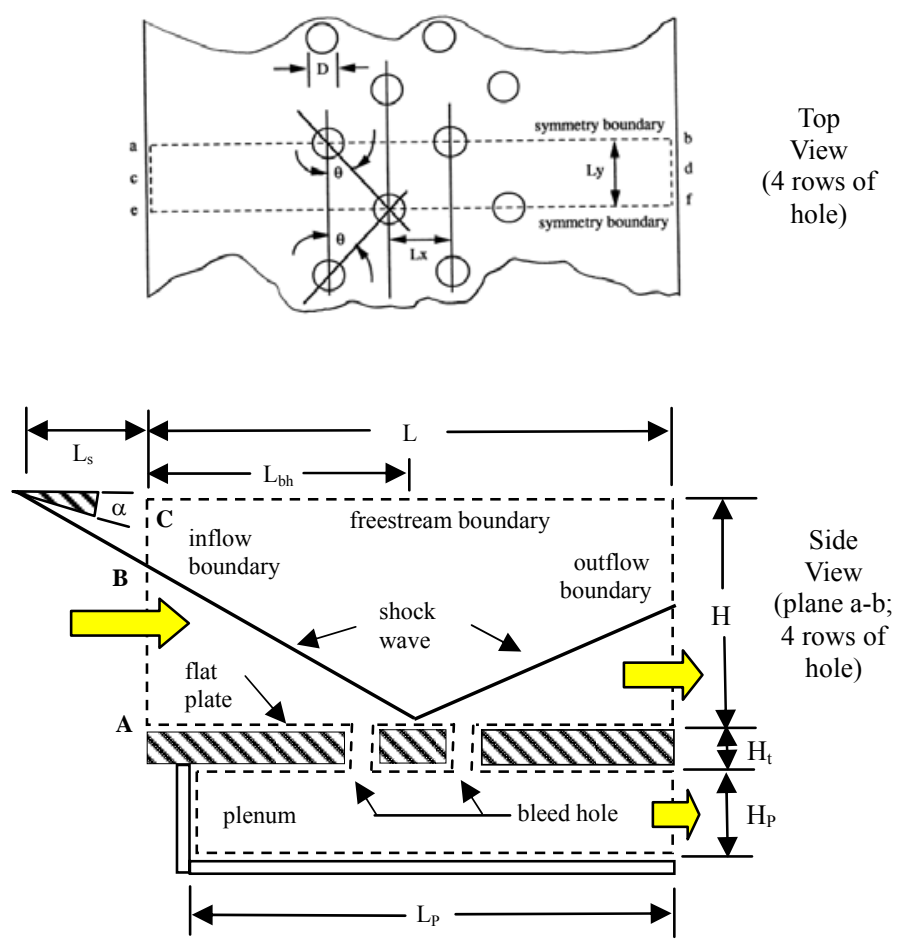

(b)

Fig. 1 Schematic of three bleed configurations.
(a) Configurations 1 (single hole) and 2 (three holes in tandem).
(b) Configuration 3 (four rows of holes).

\subsection{Problem Formulation}

The bleed problem described in the previous subsection was modeled by the density-weighted, ensemble-averaged conservation/balance equations of mass, momentum ("full compressible" Navier-Stokes), and total energy that are written in generalized coordinates and cast in strong conservation-law form. The effects of turbulence were modeled by the algebraic turbulence model of Baldwin \& Lomas [33]. Though this model may appear overly simplistic, it was found to yield results that agree reasonably well with the measurements of Willis, et al. [4, 5].

To obtain solutions to the conservation/balance equations, boundary and initial conditions are needed. The boundary conditions (BCs) employed are as follows. At the inflow boundary where the flow is supersonic everywhere except for a very small region next to the flat plate, two types of BCs were imposed (Fig. 1). Along segment A-B, all flow variables were specified at the freestream conditions except for the streamwise velocity, which had a turbulent boundary-layer next to the flat plate and the static temperature which varied in the boundary layer to maintain a constant stagnation temperature. The velocity in the turbulent boundary layer was described by the van Driest profile when $\mathrm{y}^{+}$is less than 50 and by the one-seventh power-law profile when 50 $\leq \mathrm{y}^{+} \leq \mathrm{y}^{+}(\delta)$, where $\delta$ is the boundary-layer thickness. An alternative is to use the turbulent boundary-layer profile suggested by Huang, et al. [34]. Along segment B-C, post-shock conditions based on inviscid oblique shock-wave theory were specified. These post-shock conditions were also specified along the freestream boundary. At the outflow boundary, where the reflected shock wave exited the computational domain, the flow is also mostly supersonic except for a small region next to the flat plate so that all flow variables were extrapolated. The BCs imposed at the two symmetry boundaries were zero derivatives of the dependent variables except for the velocity component normal to those boundaries, which was set equal to zero. At the exit of the 
plenum where the flow is subsonic, a back pressure $\left(\mathrm{P}_{\mathrm{b}}\right)$ was imposed, and density and velocity were extrapolated in the same manner as the variables at the outflow boundary. At all solid surfaces, the no-slip condition, adiabatic walls, and zero normalpressure gradients were imposed.

Even though only steady-state solutions were of interest, initial conditions were needed because the unsteady form of the conservation equations was used. The initial conditions employed in this study were as follows. In the region above the flat plate, the initial condition was the two-dimensional, steady-state solution for an incident and a reflected oblique shock wave on a flat plate based on inviscid oblique shock-wave theory. The streamwise velocity profile, however, was modified to give the van Driest/one-seventh power-law profile. This necessitated the density and static temperature to be modified as well in order to maintain constant stagnation temperature in the boundary layer. The initial conditions used in the bleed hole and plenum were stagnant air at constant stagnation temperature $\left(\mathrm{T}_{\mathrm{o}}\right)$ and static pressure $\left(\mathrm{P}_{\mathrm{b}}\right)$.

\subsection{Numerical Method of Solution}

Solutions to the ensemble-averaged conservation/balance equations of mass, momentum, and total energy closed by the Baldwin-Lomax algebraic turbulence model described in the previous subsection were obtained by using the OVERFLOW code developed at NASA Ames Research Center (see, e.g., Buning \& Chan [35]). The OVERFLOW code contains many algorithms. The one used in this study is as follows: All convection terms in the streamwise direction were upwind differenced by using the flux-vector splitting procedure of Steger \& Warming [36]. All convection terms in directions normal to the streamwise direction were centrally differenced in order to reduce artificial dissipation in those directions. All diffusion terms were also centrally differenced. The time-derivative terms were approximated by the Euler implicit formula. This low-order accurate formula was used because previous studies have shown that steady-state or quasi-steady-state solutions exist, and these are the ones sought here. The system of nonlinear equations that resulted from the aforementioned approximations to the space- and time-derivatives were analyzed by using the partially split method of Steger, et al. [37]. In OVERFLOW, Jacobians and metric coefficients are interpreted as grid-cell volumes and grid-cell surface areas, respectively. In this regard, all algorithms in OVERFLOW are implemented in the finite-volume manner. However, BCs in OVERFLOW are implemented in a finite-difference manner in order to enhance flexibility and ease in investigating different problems.

For the bleed configurations shown in Fig. 1 as well as their variations not shown (e.g., those with inclined holes), the computational domain was always divided into a number of zones, each with a different coordinate system in order to align upwind differencing with the streamwise direction. For this multi-zone computational domain, a chimera grid system with several overlapping grids was employed.

Figure 2 shows the grid system used for the configuration with four rows of $90^{\circ}$ holes. The grid systems used for the other configurations shown in Fig. 1 are similar. For the grid system shown in Fig. 2, the zone above the flat plate has a solutionadapted $\mathrm{H}-\mathrm{H}$ grid (adaptation is based on the initial conditions) with grid points clustered near the flat plate, bleed holes, and the impinging and reflected shock waves. The number of grid lines used is 335 from inflow to outflow, 101 from plate surface to freestream boundary, 29 or 41 between the two symmetry boundaries, depending upon the spacing between the bleed holes in the spanwise direction. The grid points closest to the flat plate have $\mathrm{y}^{+}$values less than unity. The total number of grid points in the boundary layer is about 50 . For each zone containing a bleed hole, two overlapping grids are used - an O-H grid touching the wall of the bleed hole and an $\mathrm{H}-\mathrm{H}$ grid at the center of the bleed hole. The O-H grid with $62 \times 47 \times 11$ grid points is used to resolve the circular geometry of the bleed hole. The $\mathrm{H}-\mathrm{H}$ grid with $62 \times 10 \times 15$ grid points is used to eliminate the centerline singularity associated with the $\mathrm{O}-\mathrm{H}$ grid. Note that the grids in the bleed hole overlap the grid in the plenum by four grid lines, whereas they overlap the grid above the flat plate by only two grid lines. The reason for extending the bleed-hole grids further into the plenum is to accelerate convergence rate to steady-state. For the zone containing the plenum, a single H-H grid was used ( $335 \mathrm{x} 29 \mathrm{x} 51$ or 335 x 41 x 51 depending upon the spacing between bleed holes in the spanwise direction). The grid points were clustered near all walls and bleed holes.

All grid systems used were generated by algebraic methods with one-dimensional stretching functions. Grid spacing in different grids was made comparable in regions where they overlapped in order to minimize aliasing error. The clustering and the number of grid points used in each zone were determined by numerical experiments for grid independence.

During computations, the flow field in each grid was analyzed one at a time in the following order: (i) the H-H (ii) the H-H grid in the bleed hole, (iii) the O-H grid in the bleed hole, (iv) repeat ii and iii for all bleed holes, and (v) the $\mathrm{H}-\mathrm{H}$ grid in the plenum. Information from one grid was passed to another grid via trilinear interpolation at grid boundaries. The required interpolation coefficients were obtained by using the PEGSUS code (Benek, et al. [38]). This process of analyzing the flow in one grid at a time until all grids are analyzed was repeated for each time step until a converged solution was obtained.
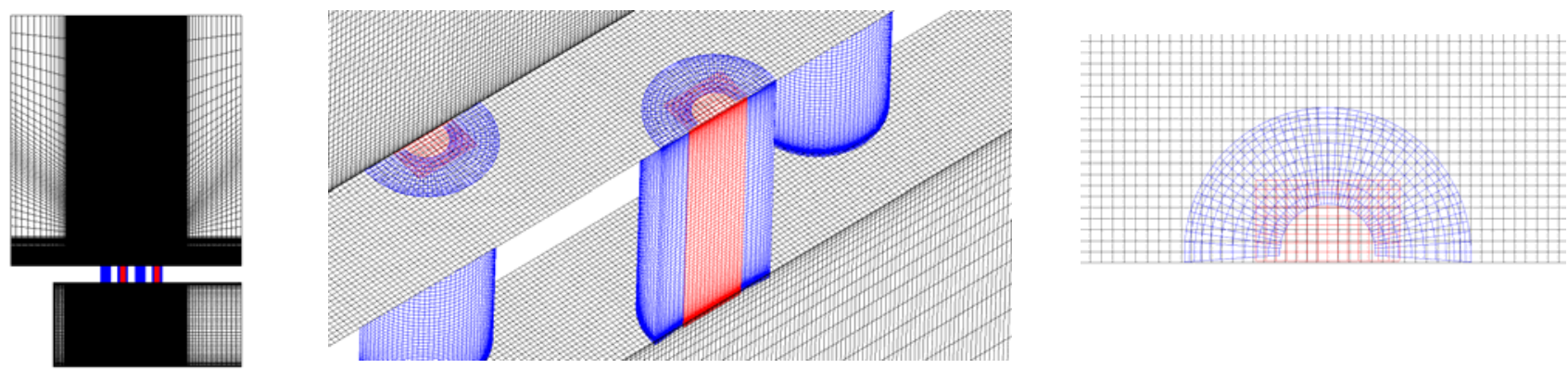

Fig. 2 Chimera grid used for the bleed problem with four rows of bleed holes. 


\section{Results}

When a shock wave impinges on a boundary layer, terminating at the sonic line, the displacement thickness of the boundary layer is increased upstream of the impingement point. This is because the adverse pressure gradient created by the incident shock propagates upstream through the subsonic portion of the boundary layer. The thickened boundary layer upstream of the incident shock causes the approaching supersonic flow to form compression waves, which coalesce to form the reflected shock wave. This incident shock, if sufficiently strong, can cause the boundary layer to separate. The purpose of bleed is to prevent this shockinduced flow separation from taking place. As noted, how bleed accomplishes this in supersonic flow conditions is not well understood. In this section, CFD results are presented for the three bleed configurations described in Section 3 to show the nature of the flow that takes place with bleed and the flow mechanisms that can be utilized to eliminate shock-induced flow separation with minimal bleed.

\subsection{Bleed from a Single Hole}

Figure 3 shows Mach contours with velocity vectors in a plane that passes through the center of a normal $90^{\circ}$ and an inclined $30^{\circ}$ bleed hole. From this figure, it can be seen that bleeding a supersonic boundary layer through a hole creates a shock structure in and around the hole. The structure of this shock first observed and described by Shih, et al. [12] is depicted in Fig. 4. The mechanism by which this shock structure forms is as follows. First, bleed causes the supersonic flow to turn towards the hole. This turning accelerates the supersonic portion of the boundary layer via the Prandtl-Meyer expansion wave. As the flow reaches the circular wall of the bleed hole, a shock structure forms because the supersonic flow must either turn downwards into the hole or turn upwards to flow along the plate. Thus, the shock structure formed is a two-segment shock system, connected by a small bow shock just upstream of wall, where the flow turning occurs. One segment is located inside the bleed hole, created by the flow that turned into the bleed hole. The other segment is formed towards the downstream edge of the bleed hole and extends well above the bleed hole, and it is created by the supersonic flow that did not enter the bleed hole but turned to flow along the plate. For an inclined hole, the strength of the shock segment inside the bleed hole may be weak or non existent since the turning required to enter the bleed hole may be minimal except next to the wedge.

To further understand the structure of the shock that forms inside the bleed hole, consider three placements of the single $90^{\circ}$ hole relative to the incident shock - upstream, at, and downstream of shock. Figure 5 shows the pressure on the flat plate from inflow to outflow about the center of the bleed hole for these three placements. Also, shown in this figure is the pressure distribution without bleed, which produced flow separation, and the exact inviscid solution with zero boundary-layer thickness. From this figure, two observations can be made. The first observation is that the pressure rise induced by the two-segment shock can be higher than the one created by the incident shock. There are two reasons for this. First, the Prandtl-Meyer expansion waves accelerated the flow which increased the flow Mach number beyond the freestream Mach number. Second, the amount of turning needed to enter into the hole or to flow along the plate can be higher than the turning angle required by the incident shock. The second observation is that the upstream influence length created by the incident shock is reduced with bleed. The reduction is greatest when the bleed hole is located slightly upstream of the incident shock. In fact, for this bleed-hole placement, there are essentially no adverse pressure gradients upstream of the bleed hole. Also, though not shown, flow separation did not take place along that plane. This indicates that the two-segment shock structure induced by bleed was able to completely block the adverse pressure gradient induced by the incident shock from propagating upstream. Hence, Shih, et al. [12] labeled this two-segment shock system induced by the bleed process as the "barrier" shock. Not shown is that the barrier shock can extend well above the boundary layer, and creates a roughness like disturbance above the plate.

The reader is referred to Shih, et al. [12] and Chyu, et al. [14] for further discussions on the structure of the barrier shock and the effects of pressure ratios across the bleed hole.

(a)

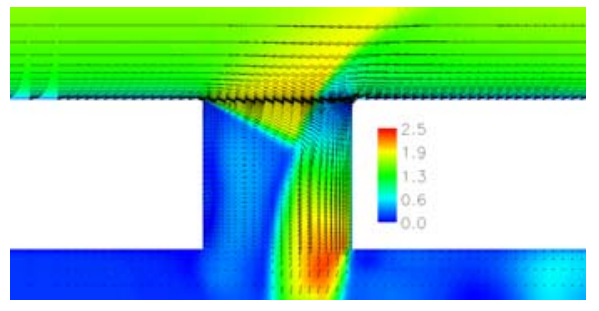

(b)

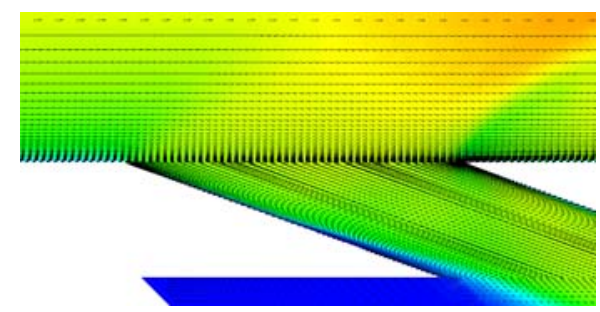

Fig. 3 Mach contours with velocity vectors in a plane that passes through the center of the bleed hole. (a) single $90^{\circ}$ hole. (b) single $30^{\circ}$ hole.

(a)

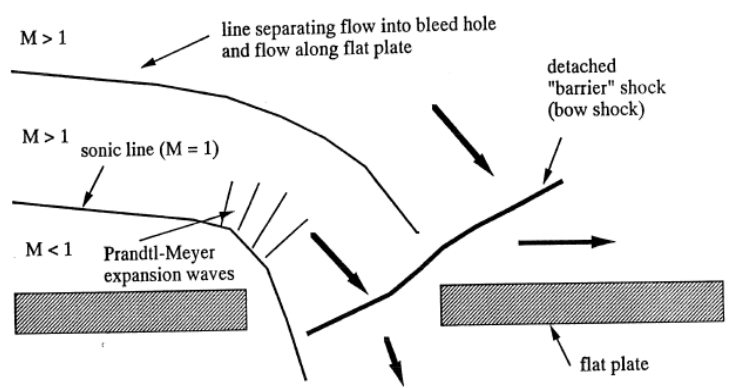

Fig. 4 Shock structure in hole induced by bleed. (b)

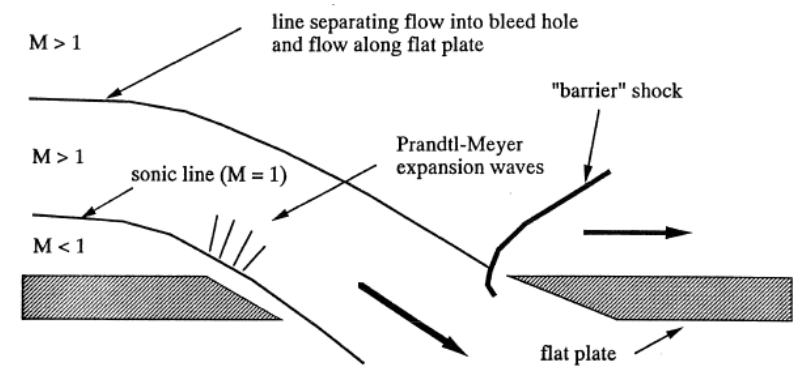

(a) Normal hole. (b) Inclined hole. 


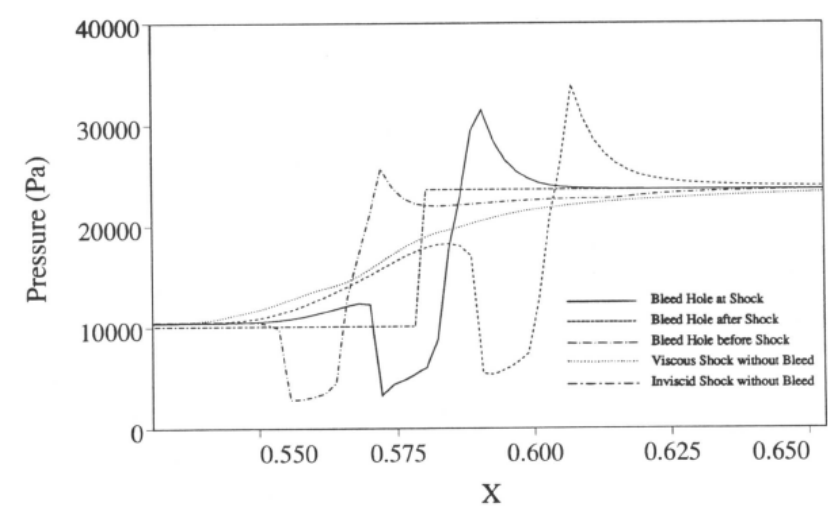

Fig. 5 Pressure along plate through center of $90^{\circ}$ hole.

(a)

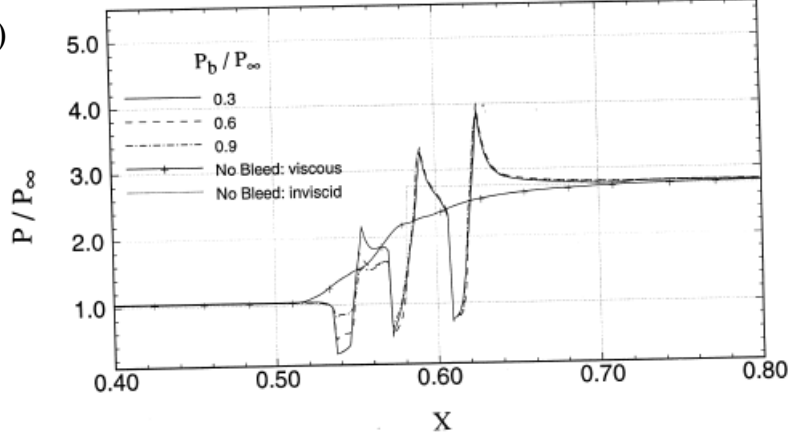

(b)

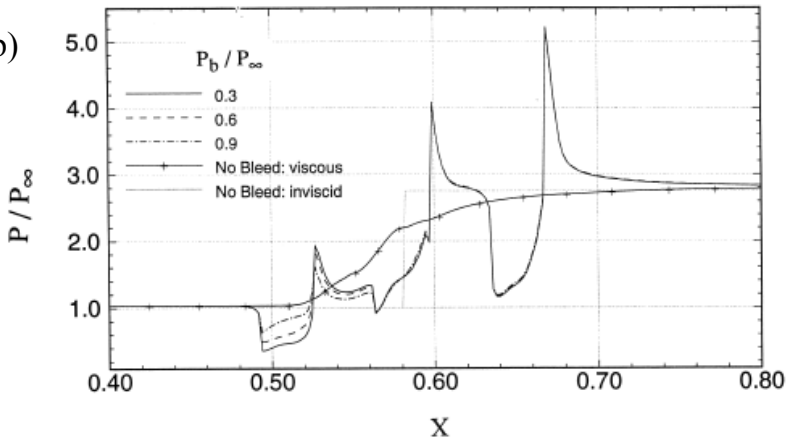

(a) $90^{\circ}$ holes. (b) $30^{\circ}$ holes. (a)

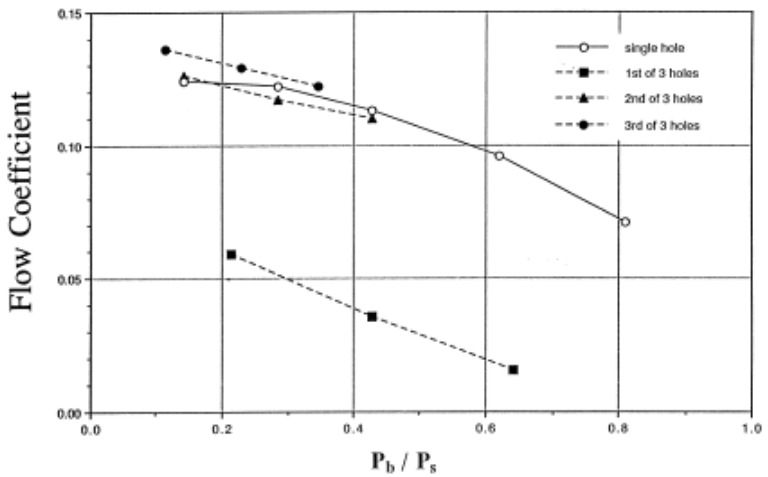

(b)

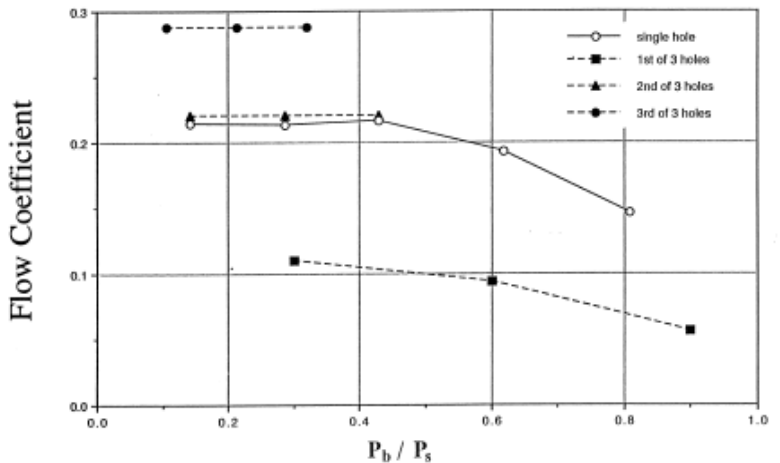

Fig. 7 Flow coefficient as a function of pressure ratio for the three-hole configuration. (A) $90^{\circ}$ holes. (B) $30^{\circ}$ holes

\subsection{Bleed from Three Holes in Tandem}

Figures 6 and 7 show the effects of bleeding a supersonic boundary layer through three holes in tandem with the incident shock wave impinging on the third hole. Figure 6 shows the pressure distribution from inflow to outflow passing through the center of the bleed holes, and Fig. 7 shows the flow coefficients as a function of the normalized back pressure $\left(\mathrm{P}_{b} / \mathrm{P}_{\mathrm{s}}\right)$, where $\mathrm{P}_{\mathrm{s}}$ is the average static pressure over the bleed hole when there is no bleed. Note that $\mathrm{P}_{\mathrm{s}}$ has a different value over different bleed holes because of the incident shock. The flow coefficient is defined as the actual bleed rate divided by the ideal bleed rate with the ideal bleed rate being sonic flow through the entire bleed hole at freestream stagnation temperature and pressure.

From Fig. 6, it can be seen that when there are three bleed holes in tandem, whether $90^{\circ}$ or $30^{\circ}$, there are no adverse pressure gradients on the flat plate. All adverse pressure gradients take place over the bleed holes, where they cannot cause flow separation.

In Fig. 7, only the second (middle) hole can be compared with the single hole case because the location is the same with respect to the incident shock. From this figure, it can be seen that the flow coefficient of the second hole is nearly the same as that from a single hole for both $90^{\circ}$ and $30^{\circ}$ holes. Thus, the influence of the upstream and downstream holes is not significant. Also, it can be seen that when there are three holes, the flow coefficient through each hole can differ significantly from each other with the difference for $30^{\circ}$ holes greater than those for $90^{\circ}$ holes. The increase in flow coefficient and hence bleed rate can be attributed to three reasons. The first is due to the rise in density, static pressure, and static temperature created by the incident shock. The second is due to the rise in density, static pressure, and static temperature created by the preceding barrier shocks. The third is due to bleed in the preceding holes, which brought fluid with higher stagnation pressure and temperature closer to the surface to be bled by the next hole.

For further discussion on the effects of holes in tandem, the reader is referred to Chyu, et al. [14]. 


\subsection{Bleed from Rows of Holes}

Figure 8 shows the pressure on the flat plate along several lines (see Fig. 1(b) for definition of a-b, c-d, and e-f). From Fig. 8(a), it can be seen that even without an incident shock, the bleed process creates a barrier shock in each bleed hole. All barrier shocks have similar structure. However, the strength of the shock in rows 3 and 4 are slightly stronger than those in rows 1 and 2 . Not shown is that the barrier shock in each hole sets up considerable disturbances in the flow above the flat plate that extend well beyond the thickness of the approaching boundary layer.

With an incident shock, the structure of the flow upstream of that shock is almost identical to those for the case without the incident shock (Fig. 8). Downstream of where the incident shock impinges on the boundary layer (which is slightly upstream of the bleed holes in the third row), the Mach number and pressure contours begin to differ considerably from the case without the incident shock. The most pronounced differences are as follows. First, barrier shocks in holes located in rows 3 and 4 are much stronger than those in rows 1 and 2 for obvious reasons. Second, the jets issuing through holes in rows 3 and 4 penetrated much deeper into the plenum than those in rows 1 and 2. This is because the incident shock increased the amount of bleed by increasing the density and static temperature of the flow above the flat plate and by reducing the size of the separation bubble in the bleed holes. Third, jets issuing through bleed holes in rows 3 and 4 interact. These interactions affect the structure of the jet in the bleed hole and in the plenum and hence the bleed rate. At this point, it is interesting to note that despite the aforementioned differences, the disturbances in the flow above the plate created by bleed with and without the incident shock appear to be of similar magnitude.

Figure 9 shows the effects of the angle $\theta$ and $L_{y}$ (Fig. 1(c)) on shock-wave/boundary-layer interactions with bleed. From this figure, it can be seen that when holes are arranged in a staggered fashion, they become connected to each other in exerting considerable spanwise influence. In particular, two rows of bleed holes arranged in a staggered fashion were able to prevent the adverse pressure gradient from propagating upstream for the three cases simulated. Even when spacing between holes in the spanwise direction was big enough so that pressure waves should be able to propagate upstream in the space between the holes (e.g., $\theta=60^{\circ} \& \mathrm{~L}_{\mathrm{y}}=1.5 \mathrm{D}$ ), most of the adverse pressure gradients induced by the incident shock were blocked. This indicates that only two rows of bleed holes are needed to control shock-wave induced flow separation. But, those two rows must be arranged in a staggered fashion and placed slightly upstream of where the incident shock wave impinges on the sonic line of the supersonic boundary layer. The spacing between the holes in the streamwise and spanwise directions can be optimized to minimize the amount of bleed needed to control separation.

(a)

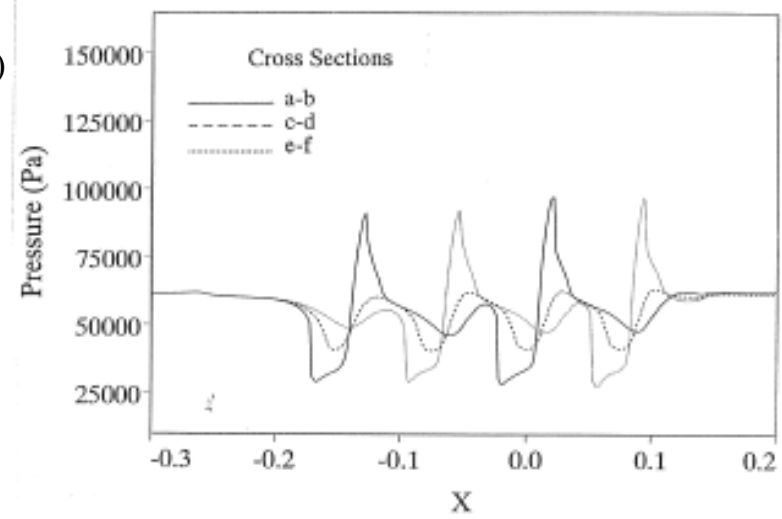

(b)

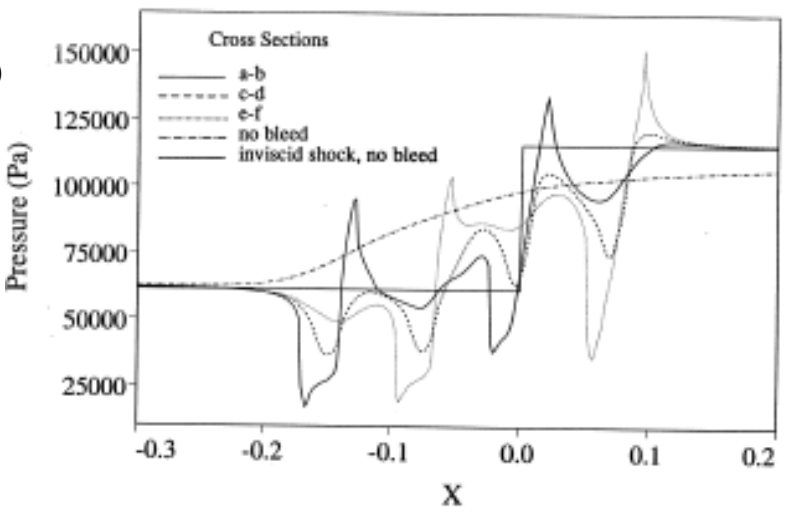

Fig. 8 Pressure distribution along plate $\left(\theta=60^{\circ}, L_{y}=D\right)$. (a) No incident shock. (b) With incident shock.

(a)

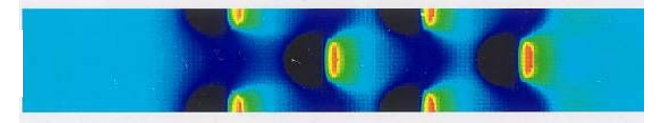

(b)

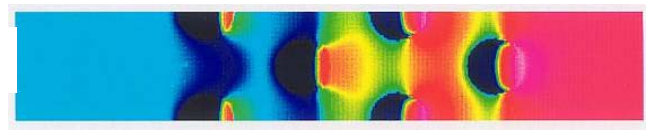

(c)
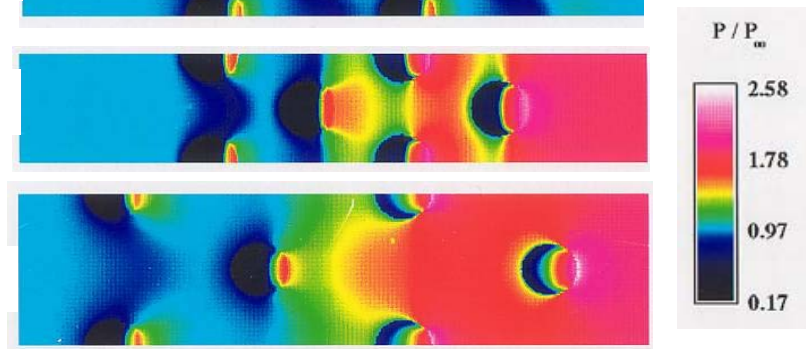

(d)

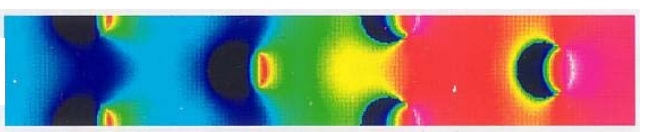

Fig. 9 Pressure on the surface of flat plate. (a) $\theta=60^{\circ} \& \mathrm{~L}_{\mathrm{y}}=\mathrm{D}$; no incident shock. $\quad$ (b) $\theta=60^{\circ} \& \mathrm{~L}_{\mathrm{y}}=\mathrm{D}$ with incident shock. (c) $\theta=60^{\circ} \& \mathrm{~L}_{\mathrm{y}}=1.5 \mathrm{D}$ with incident shock. (d) $\theta=70^{\circ} \& \mathrm{~L}_{\mathrm{y}}=\mathrm{D}$ with incident shock. 


\section{Summary}

This paper surveyed research on shock-wave/boundary-layer interaction on a flat plate with bleed through circular holes performed since 1991. This paper also described CFD studies aimed at understanding the shock structure created by the bleed process with and without an incident shock wave and how that structure can be utilized to control shock-wave-induced flow separation.

\section{Acknowledgments}

This work was supported by grant NAG 2-549 and NCC 2-845 from NASA Ames Research Center and grant NAG 3-1994, NAG 3-2234, and NAG 3-2576 from NASA Glenn Research Center. The author is grateful for this support. The author is also grateful to his co-workers - W.J. Chyu of NASA Ames and Brian Willis and David Davis of NASA Glenn - and his students Thomas Hahn, Mark Rimlinger, Yu-Liang Lin, Mark Stephens, Andrew Flores, and Dave Benson - whose work is summarized in this review.

\section{References}

[1] Seddon, J. and Goldsmith, E.L., 1985, Intake Aerodynamics, AIAA, New York.

[2] Delery, J.M., 1885, "Shock Wave / Turbulent Boundary Layer Interaction and Its Control," Progress in Aerospace Sciences, Vol. 22, pp. 209-280.

[3] Hamed, A. and Shang, J., 1991, "Survey of Validation Data Base for Shockwave Boundary Layer Interactions in Supersonic Inlets," AIAA Journal of Propulsion and Power, Vol. 7, No. 4, pp. 617-625.

[4] Willis, B.P., Davis, D.O., and Hingst, W.R., 1995a, "Flow Coefficient Behavior for Boundary Layer Bleed Holes and Slots," AIAA Paper 95-0031.

[5] Willis, B.P., Davis, D.O., and Hingst, W.R., 1995b, "Flowfield Measurements in a Normal-Hole-Bled Oblique Shock-Wave and Turbulent Boundary-Layer Interaction," AIAA 95-2885.

[6] Hamed A. and Lehnig, T., 1992, "Investigation of Oblique Shock/Boundary Layer/Bleed Interaction," AIAA Journal of Propulsion and Power, Vol. 8, No. 2, pp. 418-424.

[7] Hamed, A. and Lehnig, T., 1995, "Effect of Bleed Configuration on Shock/Boundary Layer Interactions," AIAA Journal of Propulsion and Power, Vol. 11, No. 1, pp. 42-48.

[8] Hamed, A., Shih, S.H., and Yeuan, J.J., 1992, "An Investigation of Shock/Turbulent Boundary Layer/Bleed Interactions," AIAA Paper 92-3085.

[9] Hamed, A., Shih, S.H., and Yeuan, J.J., 1993, “A Parametric Study of Bleed in Shock Boundary Layer Interactions," AIAA Paper 93-0294.

[10] Hahn, T.O., Shih, T.I-P., and Chyu, W.J., 1993, "Numerical Study of Shock-Wave / Boundary-Layer Interactions with Bleed," AIAA Journal, Vol. 31, No. 5, pp. 869-876.

[11] Rimlinger, M.J., Shih, T. I-P., and Chyu, W.J., 1992, "Three-Dimensional Shock-Wave / Boundary-Layer Interactions with Bleed through a Circular Hole," AIAA Paper 92-3084.

[12] Shih, T.I-P., Rimlinger, M.J., and Chyu, W.J., 1993, "Three-Dimensional Shock-Wave / Boundary-Layer Interactions with Bleed," AIAA Journal, Vol. 31, No. 10, pp. 1819-1826.

[13] Chyu, W.J., Rimlinger, M.J., and Shih, T.I-P., 1993, "Effects of Bleed-Hole Geometry and Plenum Pressure on ThreeDimensional Shock-Wave / Boundary-Layer / Bleed Interactions," AIAA Paper 93-3259.

[14] Chyu, W.J., Rimlinger, M.J., and Shih, T.I-P., 1995, "Control of Shock-Wave/Boundary-Layer Interactions by Bleed," AIAA Journal, Vol. 33, No. 7, pp. 1239-1247.

[15] Rimlinger, M.J., Shih, T.I-P., and Chyu, W.J., 1994, "Three-Dimensional Shock-Wave / Boundary-Layer Interactions with Bleed through Multiple Holes," AIAA Paper 94-0313.

[16] Rimlinger, M.J., Shih, T.I-P., Chyu, W.J., Willis, B.P., Davis, D.O., 1996, “Computations of Shock-Wave/Boundary-Layer Interactions with Bleed," AIAA Paper No. 96-0432.

[17] Rimlinger, M.J., Shih, T.I-P., and Chyu, W.J., 1996, "Three-Dimensional Shock-Wave / Boundary-Layer Interactions with Bleed through Rows of Holes," AIAA Journal of Propulsion and Power, Vol. 12, No. 2, pp. 217-224.

[18] Flores, A.J., Shih, T.I-P., Davis, D.O., and Willis, B.P., 1999, "Bleed of Supersonic Boundary-Layer Flow through Rows of Normal and Inclined Holes," AIAA Paper 99-2112.

[19] Lin, Y.-L., Stephens, M.A., Shih, T.I-P., and Willis, B.P., 1997 "Effects of Plenum Size on Bleeding a Supersonic Boundary Layer," AIAA Paper 97-0609.

[20] Lin, Y.-L., Shih, T.I-P., and Willis, B.P., 1997a, “Control of Shock-Wave/Boundary-Layer Interactions with Passive Blowing and Bleeding," AIAA Paper 97-3002.

[21] Chyu, W.J., Rimlinger, M.J., and Shih, T.I-P., 1995, "A Procedure for Automating CFD Simulations of an Inlet-Bleed Problem," NASA Workshop on Surface Modelling, Grid Generation, and Related Issues in CFD Solutions," NASA CP-3291, pp. 731-749.

[22] Shih, T. I-P., Lin, Y.-L., Flores, A.J., Stephens, M.A., Rimlinger, M.J., and Willis, B.P., 1998, “An Automated CFD Design and Analysis Tool for Inlet-Bleed Systems," ASME DETC98/CIE-6033.

[23] Flores, A.J., Amon, C.H., Shih, T.I-P., Davis, D.O., and Willis, B.P., 1999a, "Boundary-Layer Bleed through Micro Holes," AIAA Paper 99-0880.

[24] Shih, T.I-P., Benson, T.J., Willis, B.P., Rimlinger, M.J., and Chyu, W.J., 1997, "Structure of Shock-Wave / Boundary-Layer Interaction with Bleed through Rows of Circular Holes," AIAA Paper 97-0508. 
[25] Chyu, W.J., Howe, G.W., and Shih, T.I-P., 1992, "Bleed Boundary Conditions for Numerically Simulated MixedCompression Supersonic Inlet Flows," AIAA Journal of Propulsion and Power, Vol. 8, pp. 862-868.

[26] Smeltzer, D.B. and Sorensen, N.E., 1972, “Test of a Mixed Compression Axisymmetric Inlet with Large Transonic Mass Flow at Mach Numbers 0.6 to 2.65," NASA TN D-6971.

[27] Harloff, G.J. and Smith, G.E., 1995, “On Supersonic-Inlet Boundary-Layer Bleed Flow,” AIAA Paper 95-0038.

[28] Paynter, G.C., Treiber, D.A., and Kneeling, W.D., 1994, "Modelling Supersonic Inlet Boundary Layer Bleed Roughness," AIAA Journal of Propulsion and Power, Vol. 9, No. 4, pp. 622-627.

[29] Lee, J., Sloan, M.L., and Paynter, G.C., 1994, “A Lag Model for Turbulent Boundary Layers Developing over Rough Bleed Surfaces," AIAA Journal of Propulsion and Power, Vol. 10, No. 4, pp. 562-568.

[30] Benson, D., Shih, T.I-P., Davis, D.O., and Willis, B.P., 2000, "Boundary Conditions for CFD Simulations of Supersonic Boundary-Layer Bleed through Discrete Holes," AIAA Paper 2000-0888.

[31] Benson, D.B., Shih, T.I-P., Davis, D.O., and Willis, B.P., 2001, "Bleed Boundary Conditions for CFD Simulations of Supersonic Flows with Embedded Shocks and Boundary-Layer Bleed," ASME Paper FEDSM-2001-18116.

[32] Benson, D.B, Shih, T.I-P., and Davis, D.O., 2003, "CFD Simulations of an Axisymmetric Mixed-Compression Inlet with Bleed through Discrete Holes," ASME Paper FEDSM 2003-45076.

[33] Baldwin, B. and Lomax, H., "Thin Layer Approximation and Algebraic Model for Separated Turbulent Flows," AIAA Paper 78-257, 1978.

[34] Huang, P.G., Bradshaw, P., and Coakley, T.J., 1993, "Skin Friction and Velocity Profile Family for Compressible Turbulent Boundary Layers," AIAA Journal, Vol. 31, No. 9, pp. 1600-1604.

[35] Buning, P.G. and Chan, W.M. (1991), "OVERFLOW/F3D User's Manual," NASA - Ames Research Center.

[36] Steger, J.L. and Warming, R.F., 1981, "Flux-Vector Splitting of the Inviscid Gasdynamic Equations with Application to Finite-Difference Methods,” Journal of Computational Physics, Vol. 40, No. 2, 1981, pp. 263-293.

[37] Steger, J.L., Ying, S.X., and Schiff, L.B., 1986, “A Partially Flux-Split Algorithm for Numerical Simulation of Compressible Inviscid and Viscous Flow," Proceedings of the Workshop on Computational Fluid Dynamics, Institute of Nonlinear Sciences, University of California, Davis, California.

[38] Benek, J.A., Buning, P.G., and Steger, J.L., 1985, “A 3-D Chimera Grid Embedding Technique,” AIAA Paper 85-1523. 